\title{
Arterial Hemodynamics in Human Hypertension
}

\author{
C. T. Ting, * Kenneth P. Brin,‡ S. J. Lin," S. P. Wang," M. S. Chang," \\ Benjamin N. Chiang," and Frank C. P. Yin $\neq \$$ \\ *Department of Medicine, Veterans General Hospital, Taichung, Taiwan, Republic of China; $\ddagger$ Department of Medicine, \\ and §Department of Physiology, Johns Hopkins Medical Institutions, Baltimore, Maryland 21205; "Department of Medicine, \\ Veterans General Hospital, Taipei, Taiwan, Republic of China
}

\begin{abstract}
Differences in aortic impedance between normotensives and hypertensives are not well characterized. We examined impedance in 8 normotensive and 11 hypertensive (mean 96.7 vs. 122.2 $\mathrm{mmHg}$ ) age-matched, Chinese patients undergoing cardiac catheterization at rest, during nitroprusside, and handgrip exercise before and after beta blockade (propranolol). Hypertensives had higher resistance $\left(2,295\right.$ vs. $\left.1713 \mathrm{dyn}-\mathrm{s} / \mathrm{cm}^{5}\right)$, characteristic impedance (145.7 vs. $\left.93.9 \mathrm{dyn}-\mathrm{s} / \mathrm{cm}^{5}\right)$, total external power $(1,579$ vs. $1174 \mathrm{~mW}$ ), peripheral reflections (ratio of backward to forward wave components of 0.54 vs. 0.44 ), and first zero crossing of impedance phase angle $(4.15 \mathrm{vs} .2 .97 \mathrm{~Hz})$. These abnormalities were eliminated with vasodilatation. Differences between groups were not further exacerbated when pressure was increased during handgrip exercise. Beta blockade further increased resistance and reflections. Thus, hemodynamic abnormalities of essential hypertension (increased resistance, reflections, and pulse wave velocity, and decreased compliance) are compatible with an increased vasomotor tone that is further unmasked during generalized beta blockade.
\end{abstract}

\section{Introduction}

Arterial hypertension affects millions of people throughout the world. Vast amounts of time and money have been spent and thousands of papers have been written on better diagnosing, categorizing, and treating this affliction. In contrast, surprisingly few studies have examined in detail the hemodynamic alterations accompanying hypertension (1-4). Consequently, there are many unanswered questions regarding the relationship between cardiovascular abnormalities and hypertension, in particular: $(a)$ Why do hypertrophy and eventual cardiomyopathy often result despite seemingly adequate treatment of the elevated blood pressure? $(b)$ Are there more subtle vascular abnormalities than elevated resistance that accompany the various types of hypertension? (c) If so, what role, if any, do these abnormalities have in the natural history of the disease process? $(d)$ How are these abnormalities affected by the various classes of antihypertensive agents? $(e)$ What is the temporal sequence of the vascular abnormalities in relation to the duration, extent, or type of hypertension?

Address reprint requests to Dr. Yin, Cardiology Division, 565 Carnegie Building, The Johns Hopkins Hospital, Baltimore, MD 21205.

Received for publication 14 March 1986 and in revised form 3 July 1986.

J. Clin. Invest.

(c) The American Society for Clinical Investigation, Inc. 0021-9738/86/12/1462/10 \$1.00

Volume 78, December 1986, 1462-1471
Many studies have demonstrated that the very early phase of primary or essential hypertension is characterized by a hyperdynamic state with increased cardiac output and normal peripheral resistance (5-7). Later one finds an elevated peripheral resistance and normal or low cardiac output (8-10). The cardiac sequelae of hypertension are generally attributed to this increased load imposed on the heart. Peripheral resistance, however, is an incomplete description of the vascular properties; it describes only the nonpulsatile component of the load. Because blood flow is pulsatile, the total load comprises both pulsatile and nonpulsatile components. These load components are accounted for when the impedance concept is used to describe the arterial system $(11,12)$. Aortic impedance embodies, in addition to resistance, the summated effects of peripheral wave reflections, pulse wave velocity, and aortic stiffness and compliance. Aortic impedance thus has been found to be abnormal when vascular properties in humans are altered by aging (13), heart failure (14$17)$, coronary artery disease (3), and some physiological maneuvers $(18,19)$.

Consequently, if there are significant vascular abnormalities associated with hypertension, aortic impedance in hypertensive subjects should also be abnormal. The few studies describing the alterations in aortic impedance with hypertension, however, have produced conflicting results. O'Rourke $(1,2)$ suggested that hypertension would increase peripheral resistance, characteristic impedance, and wave reflections, and cause a shift to the right of the impedance spectrum, changes that increase both the steady and pulsatile components of the external work. However, very little data from hypertensive animals or humans were presented to substantiate these predictions. Merillon et al. (4) found that compared with normal subjects those with hypertension had greater resistance, wave reflections, and fraction of pulsatile power, and an impedance spectrum further to the right but no difference in characteristic impedance. In contrast, Nichols et al. (3) found that characteristic impedance also was greater in hypertensive as compared with normotensive patients with coronary artery disease. In both of these studies the hypertensive patients were significantly older than the control group and patients in the latter study may also have had peripheral atherosclerotic disease. Consequently, the independent effect of hypertension on aortic impedance cannot be assessed from existing studies.

The purpose of this study was to characterize differences in aortic impedance accompanying essential hypertension without the confounding factors of different ethnic backgrounds, age, or the presence or absence of atherosclerosis. We thus compared the resting aortic impedances in age-matched normotensive and hypertensive Chinese patients, all of whom were likely to be free of atherosclerosis. Moreover, to see if the differences in resting hemodynamics would be exaggerated during acute elevation in blood pressure, we compared the acute response to isometric handgrip exercise. To investigate the influence of smooth muscle 
tone on resting hemodynamics, we also measured the impedance in the hypertensive group after vasodilatation with nitroprusside. Finally, to test the hypothesis that beta adrenergic blockade results in further exacerbation of the hemodynamic responses we administered propranolol and repeated the resting and isometric exercise in the hypertensive group.

\section{Methods}

Patient selection. Candidates for the study were ethnic Chinese who were undergoing diagnostic cardiac catheterization for chest pain syndrome, evaluation of a systolic murmur, or electrophysiologic study for paroxysmal supraventricular tachycardia. The normotensive group was selected from those patients who had no prior history of symptoms related to hypertension, who had normal physical examinations, and who had multiple outpatient blood pressures that were consistently in the normal range (systolic $<140$ and diastolic $<90 \mathrm{mmHg}$, respectively). The hypertensive group was selected from a population with recently diagnosed hypertension (multiple measurements of systolic and diastolic pressures $>140$ and 90 , respectively). To avoid including patients with secondary hypertension, the hypertensive group included only those patients who had a normal rapid sequence intravenous pyelogram, adrenal echogram, and renal arteriogram. In addition all subjects had normal serum levels of electrolytes, creatinine, urea nitrogen, aldosterone, triiodothyronine $\left(\mathrm{T}_{3}\right)$, and thyroxine $\left(\mathrm{T}_{4}\right)$; normal creatinine clearance; and normal urinary excretion levels of electrolytes, vanillylmandelic acid, 17-hydroxycorticosterone, and 17-ketosteroids. All patients gave informed consent for the investigative portion of the study.

Catheterization. All studies were performed after premedication with Benadryl, $50 \mathrm{mg}$ i.m. Both right- and left-heart catheterizations were performed using the percutaneous approach via a femoral vein and artery, respectively. The diagnostic catheterization was completed first, and only those patients with no evidence of hemodynamically significant coronary heart disease ( $<50 \%$ narrowing of any major coronary artery), congenital heart disease, or valvular heart disease were entered into the study.

The methodology for the catheterization and data acquisition was identical to that reported earlier (16) and will be briefly summarized here. After completion of the diagnostic portion of the study, the standard left-heart catheter was replaced by a specially designed high-fidelity transducer catheter (model VPC 673-D, Millar Instruments Co., Houston, TX). This 7F arterial catheter had two pressure transducers located at 6 $\mathrm{cm}$ from the tip. In addition there was an electromagnetic flow velocity sensor located another $3 \mathrm{~cm}$ distal to the second pressure sensor. The velocity sensor was connected to a flowmeter (model BL-613 or model BL 610, Biotronex Laboratories, Kensington, MD). The flow system had a frequency response that was decreased by $3 \mathrm{db}$ at $\sim 75 \mathrm{~Hz}$. The catheter tip was advanced retrograde across the aortic valve to help stabilize the catheter and to keep the sensors in the center of the stream while allowing simultaneous measurement of left ventricular pressure and ascending aortic pressure and flow velocity. After crossing the valve, the catheter was manipulated to obtain an optimal flow velocity signal characterized by a steady diastolic level with maximal systolic amplitude and minimal late systolic negative flow (16).

The pressure and flow velocity signals during each experimental condition were recorded on analogue tape (3968-A, Hewlett-Packard Co., Waltham, MA) for later offline analysis. The signals were also monitored during the study with both a six-channel monitor (Hewlett-Packard Co.) and a strip-chart recorder. An estimation of ascending aortic cross-sectional area during each stage of the study was obtained from two-dimensional echocardiograms. To minimize drift, before inserting each catheter we soaked and prewarmed the sensors for at least $3 \mathrm{~h}$ in saline at $37^{\circ} \mathrm{C}$. After withdrawing the catheters from each patient they were reimmersed in the bath to check for baseline drift. The pressure reading at the completion of the study, with the pressure sensor barely submerged in the fluid at atmospheric pressure was used as the zero pressure reference signal.
Protocol. Baseline, resting hemodynamics were first recorded in both groups. This measurement was taken at least $30 \mathrm{~min}$ after the last injection of contrast medium. To see if the differences in the baseline hemodynamics between the two groups could be exacerbated by a rapidly reversible increase in blood pressure we asked the subjects in both groups to perform isometric handgrip exercise. Pressure, flow, and electrocardiogram (ECG) signals were recorded during a 50\% maximal voluntary contraction (MVC) $)^{1}$ for $1 \mathrm{~min}$. The MVC was determined by taking the average of the tensions obtained by three brief maximal contractions prior to beginning the investigative phase of the study. This level of handgrip has been previously shown to produce an increase in blood pressure and heart rate in both normal and hypertensive individuals without increasing peripheral resistance $(20,21)$.

To assess the role of vasomotor tone on impedance in the hypertensive group we used two interventions, first to decrease and then to increase vasomotor tone. We first examined the response during generalized vasodilatation produced by nitroprusside. Sodium nitroprusside was infused intravenously beginning at a dose of $0.25 \mu \mathrm{g} / \mathrm{kg}$ per min. After $3 \mathrm{~min}$ at each infusion level, the hemodynamic response was recorded. The dose was then doubled and recordings made after another $3 \mathrm{~min}$. The infusion was increased until the peak systolic blood pressure was consistently below $140 \mathrm{mmHg}$.

After all pressures had returned to the original levels or after $30 \mathrm{~min}$, we examined the response to an increased vasomotor tone. Previous studies have indicated that some patients with essential hypertension have an apparently increased vasomotor tone (22-27). In the final phase of the study, we thus tested the hypothesis that nonspecific beta adrenergic blockade might produce a deleterious hemodynamic effect by unmasking an increased underlying level of vasoconstriction. Propranolol was administered to the hypertensive group at a rate of $1 \mathrm{mg} / \mathrm{min}$ until a full blocking dose of $0.15 \mathrm{mg} / \mathrm{kg}$ had been delivered. Resting hemodynamic measurements were made during beta blockade. Finally, handgrip exercise was repeated to examine the pattern and the extent of the hydraulic load imposed in the setting of beta blockade in these subjects.

Calculations and data analysis. Details of the data analysis have been reported previously (16) and will only be summarized here. The analogue records were digitized at a rate of $250 \mathrm{~Hz}$ using a 12-bit analogueto-digital converter (Tecmar Labmaster, Scientific Solutions, Inc., Solon, $\mathrm{OH}$ ) interfaced to a microcomputer (IBM/PC, IBM Corp., Danbury, CT). The digitized signals were analyzed on a minicomputer (MV/8000, Data General Corp., Westboro, MA) using software we developed previously. The digitized flow velocity signals were displayed on the terminal screen and only beats that had no significant baseline drift and no significant negative dip or secondary rise in diastole were considered acceptable for analysis. Zero flow was assumed to be that in late diastole. The calibration of the flow velocity probe was performed in the majority of patients by the Fick method based on the oxygen consumption measured in the resting state immediately prior to catheterization. From the digitized flow velocity signal we determined a time-averaged flow velocity for at least three separate beats. This mean velocity was converted to volume flow by multiplying by the resting aortic cross-sectional area. The appropriate calibration factor for each probe was then determined by matching the cardiac outputs obtained from the Fick method with the mean output calculated from the digitized flow signals. In a few patients in whom the cardiac outputs were not available, a calibration factor obtained by averaging the data for that particular probe was used.

The noise level of the flow signal was determined for each patient by performing Fourier analysis on the diastolic portions of the flow signal. Only flow harmonics with moduli greater than twice the maximum noise level were included in the subsequent calculations. For acceptable beats, the pressure and flow signals were resolved into their Fourier harmonics, and the input impedance modulus and phase angle for each harmonic were calculated as the ratio of the pressure to flow moduli and the difference of the pressure and flow phase angles, respectively. We had previously determined that the combined flow-sensor flowmeter system had

1. Abbreviations used in this paper: MVC, maximal voluntary contraction. 
a phase lag of $1.3 / \mathrm{Hz}$, which was accounted for in subsequent calculations. The characteristic impedance $(\mathrm{Zc})$ was estimated by averaging the suitable impedance moduli for frequencies of $4 \mathrm{~Hz}$ and higher $(16,17)$. Total external power, consisting of both pressure and kinetic terms for the left ventricle, was calculated as previously reported (16). The oscillatory power, the steady power, and the ratio of oscillatory to total power, indicating the efficiency with which the pulsatile energy was converted into forward flow, were also calculated.

We wished specifically to compare the wave reflection properties of the hypertensive and normal groups. We have previously shown that the reflection characteristics of the arterial tree can be adequately described by calculating the ratio of the backward to the forward component of the pressure wave (28). This index of reflections is easier to analyze and compare than the more complete but cumbersome reflection spectrum. We thus decomposed the pressure wave into its forward and backward components as described previously (29) and used the ratio of the backward to forward wave to index the magnitude of the wave reflection seen in the ascending aorta during each condition.

Statistical analysis. Because the number of subjects was small and there was considerable scatter in some of the derived parameters, we wished to avoid having these "outlying" data points unduly influence the statistical analyses. At the same time, we wanted to avoid having to delete any of the data points based on arbitrary criterion. Rather than using a standard $t$ test for unpaired data with the outliers excluded based on an arbitrary criterion, we thus employed two different statistical tests for significant differences in the resting data between the two groups. We first employed biweight robust regression (30) to estimate and compare the means of the two groups. Robust regression takes the scatter of the data into account and automatically calculates a smooth weighting func- tion that gives more weight to observations near the median and less weight to outlying observations (it is possible for extreme outliers to receive zero weight). This method enables one to arrive at more statistically reliable estimates of the group means and significance of the differences than with either the raw means or the means obtained with outliers accounted for in a more arbitrary manner. We next employed the nonparametric Mann-Whitney rank test, which does not require an assumption about the specific distribution of the data (31). This test is also more appropriate than the standard $t$ test when there is wide scatter in the data (31).

Since each intervention was immediately preceded by a control state, the effect of an intervention within each group was analyzed by using paired $t$ tests. The effect of an intervention between the two groups was tested by using unpaired $t$ tests on the individual changes in each group. Differences were considered significant at the $P=0.05$ level.

\section{Results}

69 patients were entered into the study ( 58 hypertensive and 11 normotensive). Of these, 19 patients whose data met our criteria of suitability for analysis constituted the study groups. The normotensive group consisted of seven men and one woman, aged 30 to $56 \mathrm{yr}$ (mean age of $42 \mathrm{yr}$ ). The hypertensive group consisted of six men and five women with essential hypertension aged 25$53 \mathrm{yr}$ (mean age of $35 \mathrm{yr}$ ). Most of the hypertensive patients (9 of 11) were newly diagnosed and had never been treated with any antihypertensive medication. The other two had stopped using these drugs for at least 3 wk before the investigation.

Table I. Characteristics of the Study Population

\begin{tabular}{|c|c|c|c|c|c|c|}
\hline Patient & Age & Sex & Height & Weight & Clinical diagnosis & Aortic CSA \\
\hline & $y r$ & & $\mathrm{~cm}$ & $k g$ & & $\mathrm{~cm}^{2}$ \\
\hline \multicolumn{7}{|c|}{ Normal subjects } \\
\hline N1 & 30 & $\mathbf{M}$ & 180 & 74.5 & PSVT & 6.6 \\
\hline $\mathrm{N} 2$ & 56 & $\mathbf{M}$ & 173 & 71.0 & Normal & 7.2 \\
\hline N3 & 37 & $\mathbf{M}$ & 167 & 67.5 & Normal & 5.2 \\
\hline N4 & 39 & $\mathbf{M}$ & 172 & 77.0 & Normal & 6.8 \\
\hline N5 & 40 & $\mathrm{~F}$ & 161 & 53.0 & AVND & 4.7 \\
\hline N6 & 37 & $\mathbf{M}$ & 175 & 79.5 & $<50 \%$ RCA lesion & 7.7 \\
\hline N7 & 45 & $\mathbf{M}$ & 175 & 89.5 & Normal & 12.3 \\
\hline N8 & 51 & $\mathbf{M}$ & 174 & 54.0 & Normal & 9.7 \\
\hline Mean & 41.9 & & 172.1 & 70.8 & & 7.53 \\
\hline $\mathrm{SD}$ & 8.4 & & 5.8 & 12.5 & & 2.46 \\
\hline \multicolumn{7}{|c|}{ Hypertensive subjects } \\
\hline $\mathrm{H1}$ & 30 & $\mathbf{M}$ & 170 & 78.0 & Hypertension & 6.6 \\
\hline $\mathrm{H} 2$ & 25 & $\mathbf{M}$ & 173 & 68.5 & Hypertension & 6.3 \\
\hline H3 & 35 & $\mathbf{M}$ & 164 & 64.0 & $\mathrm{~S} / \mathrm{p}$ right nephrectomy & 7.0 \\
\hline H4 & 34 & $\mathbf{M}$ & 170 & 74.5 & Hypertension & 6.4 \\
\hline H5 & 35 & $\mathbf{F}$ & 163 & 59.0 & Hypertension & 6.2 \\
\hline H6 & 44 & $\mathbf{F}$ & 167 & 67.0 & Hypertension & 4.8 \\
\hline $\mathrm{H7}$ & 53 & $\mathbf{M}$ & 178 & 89.0 & Hypertension & 7.6 \\
\hline H8 & 46 & $\mathbf{F}$ & 150 & 76.0 & Hypertension & 7.7 \\
\hline H9 & 30 & $\mathbf{F}$ & 149 & 69.0 & Hypertension & 5.6 \\
\hline H10 & 25 & $\mathbf{F}$ & 146 & 45.5 & Hypertension & 4.6 \\
\hline H11 & 26 & $\mathbf{M}$ & 170 & 56.0 & Hypertension & 4.7 \\
\hline Mean & 34.8 & & 163.7 & 67.9 & & 6.13 \\
\hline SD & 9.3 & & 10.7 & 11.8 & & 1.10 \\
\hline$P(\mathrm{~N}$ vs. $\mathrm{H})$ & NS & & NS & NS & & NS \\
\hline
\end{tabular}

PSVT, paroxysmal supraventricular tachycardia; AVND, atrioventricular nodal dysfunction; RCA, right coronary artery. 
Some pertinent data for all of the subjects are listed in Table I. There was no difference between the two groups in age, height, body weight, or aortic diameter. Diagnostic cardiac catheterization revealed that none of the patients had congenital heart disease and none had acquired valvular heart disease. One of the normal subjects had a history of paroxysmal supraventricular tachycardia, another had borderline atrioventricular (AV) block, and a third had a $<50 \%$ narrowing of the right coronary artery. One of the hypertensive patients had undergone previous right nephrectomy for renal calculi but had normal renal function. All of the hypertensive patients were considered to have essential hypertension on the basis of the previously described workup.

The averaged spectrum of impedance modulus and phase angle for the groups are illustrated in Fig. 1. For clarity, only the robust estimates of the mean data for each group in each frequency range are shown. In the hypertensive group compared with the normotensive group the low-frequency harmonics of the modulus were higher and there was a rightward shift of the impedance spectra. The resting hemodynamic data for each subject are listed in Table II. For completeness, both the raw mean as well as the more robust weighted group means for all the data are shown. The statistical analysis for the weighted, unpaired data are listed along with the results of the nonparametric test for significance. In addition to the higher aortic systolic, diastolic, and mean pressures in the hypertensive as compared with the normal group, there was a significantly higher left ventricular end-diastolic pressure. There was no difference in stroke volume between the two groups and heart rate tended to be higher in the normals. As a consequence of the elevated
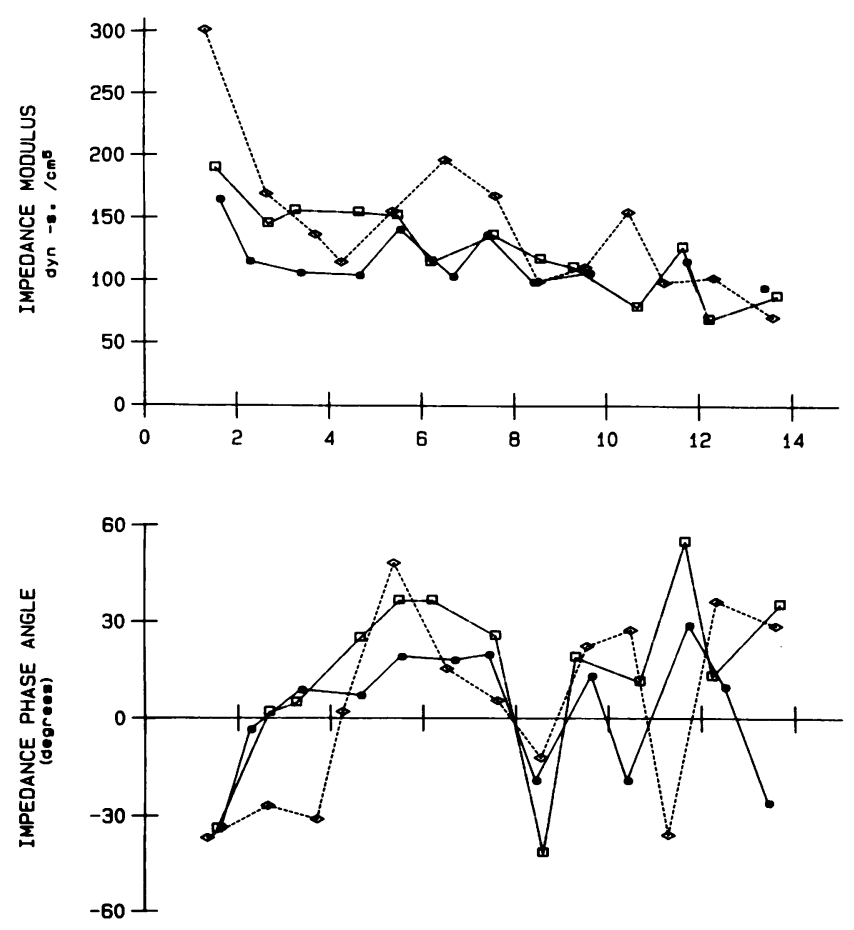

FREQUENCY (HZ)

Figure 1. Averaged resting impedance moduli and phase angle spectra for the normal subjects (solid symbols and line); for the hypertensive subjects (open diamond symbols and dotted line); and for the hypertensive subjects during nitroprusside infusion (open squares and dashdot line). pressures, the total external power tended to be greater in the hypertensive than in the normal group but the total oscillatory power and percentage of oscillatory power did not differ between the two groups. Compared with the normal subjects, the hypertensive group had greater total resistance, first modulus of impedance, characteristic impedance, and a larger ratio of the backward to forward wave. The first zero crossing of the impedance phase angle $f_{0}$, which is an index of the shift of the impedance spectra, was higher in the hypertensive group. Along with the higher resistance that is present with essential hypertension, the hemodynamic picture thus is consistent with a vasculature that has more wave reflections, a higher pulse wave velocity, and decreased compliance.

Table III compares the results in the hypertensive group during nitroprusside infusion with those of the normals at rest. Note that the data for the normals differ slightly from those presented in Table II because the group means estimated from the biweight regression depend upon the data for both groups. Except for the small but significant residual elevation of the aortic and left ventricular pressures, nitroprusside normalized all of the other hemodynamic parameters in the hypertensive group. The averaged response of this group to nitroprusside is also illustrated by the dot-dash line in Fig. 1. These data indicate that nitroprusside effectively normalizes the abnormal hemodynamics present in hypertension when the blood pressure is reduced into the normal range.

Table IV summarizes the hemodynamic findings in both groups during handgrip exercise. The upper portion of the table lists the averaged resting and exercise data along with the change in each parameter between the resting and exercise conditions for the normal subjects. The middle portion of the table lists the same data for the hypertensive group. The lower portion of the table summarizes the statistical analysis of the responses between the two groups. Exercise did not produce alterations in either group in resistance, stroke volume, percentage of oscillatory power, fraction of backward to forward waves, or in $f_{0}$. In contrast, in both groups exercise significantly increased heart rate, aortic pressures, left ventricular end-diastolic pressure, the first modulus of impedance, total and oscillatory external power, and the magnitude of the forward pressure wave. There was an increase in characteristic impedance in the normal group and in magnitude of the backward component of the pressure wave in the hypertensive group. Both these alterations were, however, quite small and probably physiologically unimportant. These data indicate that, despite the significant alteration in hemodynamics produced by exercise within each group, exercise did not exacerbate any of the hemodynamic differences between the groups beyond those that were already present at rest.

Fig. 2 illustrates the averaged impedance spectra for the hypertensive subjects before and after acute administration of intravenous propranolol. The complete hemodynamic responses are summarized in Table V. Propranolol induced small but statistically significant increases in aortic systolic, diastolic, and mean pressures. There were concomitant decreases in heart rate and total external power. Of most interest, however, was the finding that there were significant increases in resistance, in the lower frequency impedance moduli, in left ventricular end-diastolic pressure, and in both the magnitude of the reflected wave and the ratio of the backward to the forward wave. There were no alterations in stroke volume, characteristic impedance, or the magnitude or percentage of oscillatory power. Beta adrenergic blockade in this setting thus produced a further exacerbation of 
Table II. Comparison of Resting Hemodynamics in Normal and Hypertensive Subjects before Nitroprusside Infusion

\begin{tabular}{|c|c|c|c|c|c|c|c|c|c|c|c|c|c|c|c|c|}
\hline Patient & HR & SV & PAo, & PAod $_{d}$ & $\mathbf{P A o}_{\mathbf{m}}$ & LVEDP & $\mathbf{R}$ & $Z_{1}$ & $Z_{c}$ & $W_{\mathbf{t}}$ & $W_{0}$ & $W_{0} / W_{t}$ & $P_{\mathrm{f}}$ & $P_{\mathrm{b}}$ & $P_{\mathrm{b}} / P_{\mathrm{f}}$ & $f_{0}$ \\
\hline \multicolumn{17}{|c|}{ Normal subjects } \\
\hline N1 & 102.2 & 34.9 & 126.9 & 76.0 & 100.7 & 9.2 & 2288.1 & 272.1 & 238.2 & 960.0 & 156.6 & 0.16 & 45.1 & 18.1 & 0.40 & 3.51 \\
\hline N2 & 63.5 & 83.5 & 139.6 & 73.0 & 100.7 & 17.6 & 1740.5 & 234,3 & 67.6 & 1565.3 & 375.6 & 0.24 & 47.4 & 26.2 & 0.55 & 1.96 \\
\hline N3 & 89.0 & 56.9 & 106.1 & 72.2 & 88.2 & 15.3 & 1392.9 & 107.8 & 138.1 & 1159.0 & 161.4 & 0.14 & 36.9 & 14.5 & 0.39 & 2.49 \\
\hline N4 & 103.6 & 95.6 & 126.9 & 102.9 & 114.3 & 2.8 & 935.3 & 40.6 & 44.1 & 2775.6 & 261.4 & 0.09 & 24.1 & 9.0 & 0.37 & 3.07 \\
\hline N5 & 70.4 & 47.3 & 113.0 & 69.0 & 90.3 & 9.5 & 2168.3 & 242.6 & 147.3 & 805.1 & 130.9 & 0.16 & 37.7 & 16.9 & 0.45 & 2.89 \\
\hline N6 & 96.3 & 55.3 & 115.2 & 75.4 & 93.9 & 13.8 & 1434.3 & 117.7 & 94.7 & 1356.6 & 234.6 & 0.17 & 37.1 & 12.0 & 0.32 & 3.10 \\
\hline N7 & 68.8 & 63.0 & 113.5 & 80.5 & 97.2 & 7.3 & 1794.0 & 121.4 & 56.4 & 1067.0 & 127.5 & 0.12 & 40.3 & 26.3 & 0.65 & 2.92 \\
\hline N8 & 98.8 & 45.0 & 125.9 & 77.5 & 100.6 & 8.5 & 1854.9 & 181.0 & 112.6 & 1215.1 & 204.3 & 0.17 & 36.6 & 18.5 & 0.51 & 3.30 \\
\hline Mean $_{1}$ & 86.6 & 60.2 & 120.9 & 78.3 & 98.2 & 10.5 & 1701.0 & 164.7 & 112.4 & 1363.0 & 206.5 & 0.16 & 38.2 & 17.7 & 0.46 & 2.91 \\
\hline Mean $_{2}$ & 97.2 & 53.1 & 120.2 & 74.8 & 96.7 & 10.5 & 1713.3 & 165.1 & 93.9 & 1174.1 & 193.5 & 0.15 & 39.1 & 16.5 & 0.44 & 2.97 \\
\hline \multicolumn{17}{|c|}{ Hypertensive subjects } \\
\hline HI & 75.6 & 98.0 & 165.7 & 98.0 & 128.3 & 20.0 & 1379.0 & 160.0 & 113.4 & 2545.0 & 405.8 & 0.16 & 55.4 & 30.5 & 0.55 & 4.05 \\
\hline $\mathbf{H} 2$ & 83.0 & 55.3 & 142.8 & 93.6 & 118.8 & 14.6 & 2067.9 & 194.5 & 120.7 & 1369.0 & 149.9 & 0.11 & 37.6 & 21.5 & 0.57 & 4.89 \\
\hline H3 & 75.0 & 62.9 & 211.6 & 123.0 & 159.8 & 20.1 & 2721.4 & 376.7 & 154.2 & 1958.5 & 279.4 & 0.14 & 58.7 & 38.0 & 0.65 & 4.41 \\
\hline H4 & 80.6 & 81.8 & 151.8 & 92.3 & 122.1 & 11.0 & 1481.1 & 164.7 & 125.7 & 2141.3 & 343.1 & 0.16 & 52.8 & 24.3 & 0.46 & 2.26 \\
\hline H5 & 89.6 & 35.6 & 153.4 & 87.1 & 118.2 & 13.6 & 2968.1 & 425.1 & 172.5 & 1006.3 & 162.5 & 0.16 & 42.0 & 26.9 & 0.64 & 3.98 \\
\hline H6 & 76.7 & 26.9 & 160.8 & 90.9 & 121.6 & 14.5 & 4768.3 & 656.2 & 360.9 & 675.7 & 117.2 & 0.17 & 53.3 & 25.0 & 0.47 & 3.47 \\
\hline H7 & 71.9 & 62.6 & 161.9 & 90.4 & 119.7 & 14.2 & 2127.1 & 277.9 & 144.5 & 1480.0 & 278.3 & 0.19 & 52.0 & 26.7 & 0.51 & 3.86 \\
\hline H8 & 65.7 & 64.4 & 167.1 & 92.1 & 123.2 & 23.3 & 2327.4 & 362.3 & 184.8 & 1405.3 & 242.2 & 0.17 & 52.8 & 26.9 & 0.51 & 3.69 \\
\hline H9 & 82.3 & 57.6 & 206.6 & 128.0 & 162.1 & 25.9 & 2774.1 & 349.1 & 169.4 & 1986.4 & 270.7 & 0.14 & 56.6 & 29.5 & 0.52 & 4.11 \\
\hline $\mathrm{H} 10$ & 81.8 & 40.2 & 172.3 & 95.3 & 129.7 & 17.4 & 3175.2 & 518.4 & 147.9 & 1119.5 & 162.5 & 0.15 & 46.4 & 33.0 & 0.71 & 5.20 \\
\hline H11 & 85.2 & 65.2 & 148.5 & 93.5 & 121.1 & 15.3 & 1796.9 & 198.7 & 127.8 & 1765.4 & 259.1 & 0.15 & 45.5 & 19.2 & 0.42 & 4.53 \\
\hline Mean $_{1}$ & 78.9 & 59.1 & 167.5 & 98.6 & 129.5 & 17.3 & 2507.9 & 334.9 & 165.6 & 1587.0 & 242.8 & 0.16 & 50.3 & 27.4 & 0.55 & 4.04 \\
\hline Mean $_{2}$ & 79.2 & 58.4 & 158.6 & 92.5 & 122.2 & 16.7 & 2294.9 & 300.7 & 145.7 & 1578.9 & 239.1 & 0.15 & 51.2 & 26.7 & 0.54 & 4.15 \\
\hline $\mathbf{P}_{2}$ & $<.001$ & NS & $<0.001$ & $<0.001$ & $<0.001$ & $<0.01$ & $<0.05$ & $<0.025$ & $<0.01$ & $<0.05$ & NS & NS & $<0.001$ & $<0.001$ & $<0.05$ & $<0.001$ \\
\hline $\mathbf{P}_{3}$ & NS & NS & $<0.001$ & $<0.005$ & $<0.001$ & $<0.01$ & 0.025 & $<0.01$ & 0.025 & NS & NS & NS & 0.001 & 0.001 & $<0.05$ & 0.001 \\
\hline
\end{tabular}

$\mathrm{HR}$, heart rate (beats/min); SV, stroke volume (ml); $\mathrm{PAO}_{\mathrm{s}}, \mathrm{PAO}_{\mathrm{d}}, \mathrm{PAo}_{\mathrm{m}}$, systolic, diastolic, and mean aortic pressures, respectively (mmHg); $\mathrm{LVEDP,} \mathrm{left} \mathrm{ventricular}$ end-diastolic pressure $(\mathrm{mmHg}) ; R$, total arterial resistance $\left(\right.$ dyn-s $\left./ \mathrm{cm}^{3}\right) ; Z_{1}$ and $Z_{\mathrm{c}}$, first modulus of impedance and characteristic impedance, respectively $\left(\right.$ dyn-s/cm $\left.{ }^{5}\right)$; $W_{\mathrm{t}}, W_{0}$, total and oscillatory external power $(\mathrm{mW}) ; P_{\mathrm{f}}, P_{\mathrm{b}}$, magnitude of forward and backward components of pressure wave (mmHg); $P_{\mathrm{b}} / P_{\mathrm{f}}$, ratio of backward to forward components of pressure wave; $f_{0}$, first zero crossing of impedance phase angle $(\mathrm{Hz})$; mean , raw group mean; mean $_{2}$, robust weighted group mean; $P_{2}$, P-statistic from comparison of the weighted means; $P_{3}$, P-statistic from the Mann-Whitney rank test.

the already abnormal resting hemodynamics in the hypertensive patients.

Table VI summarizes the response to handgrip exercise after administration of propranolol and Table VII compares the responses to handgrip exercise before and after propranolol in the hypertensive group. The response to exercise after propranolol was somewhat different than the response to exercise prior to its administration. Both before and after administration of propranolol handgrip resulted in statistically significant increases in heart rate, aortic pressures, both forward and backward components of pressure, left ventricular end-diastolic pressure, total power, and the first modulus of impedance. Likewise, exercise in both settings resulted in no changes in percentage of oscillatory power, the ratio of backward to forward waves, or $f_{0}$. As shown in Table VII, however, compared to the prepropranolol exercise response, handgrip after propranolol produced a decrease in stroke volume. Moreover, exercise after beta blockade resulted in a larger increase in peripheral resistance and a smaller increase

Table III. Comparison of Hemodynamics in Normals and in Hypertensive Subjects during Nitroprusside Infusion

\begin{tabular}{|c|c|c|c|c|c|c|c|c|c|c|c|c|c|c|c|c|}
\hline Patient & HR & SV & PAO, $_{3}$ & PAo $_{d}$ & $\mathrm{PAO}_{\mathrm{m}}$ & LVEDP & $R$ & $Z_{1}$ & $Z_{\mathrm{c}}$ & $W_{1}$ & $W_{0}$ & $W_{0} / W_{t}$ & $P_{\mathrm{f}}$ & $P_{\mathrm{b}}$ & $P_{\mathrm{b}} / P_{\mathrm{f}}$ & $f_{0}$ \\
\hline \multicolumn{17}{|c|}{ Normal subjects $(n=8)$} \\
\hline $\operatorname{Mean}_{1}$ & 86.6 & 60.2 & 120.9 & 78.3 & 98.2 & 10.5 & 1701.0 & 164.7 & 112.4 & 1363.0 & 206.5 & 0.16 & 38.2 & 17.7 & 0.46 & 2.91 \\
\hline $\operatorname{Mean}_{2}$ & 88.0 & 57.3 & 119.8 & 74.8 & 96.7 & 10.0 & 1716.2 & 165.2 & 100.4 & 1170.3 & 193.5 & 0.15 & 39.3 & 15.7 & 0.42 & 3.02 \\
\hline \multicolumn{17}{|c|}{ Hypertensives subjects during nitroprusside $(n=11)$} \\
\hline Mean $_{1}$ & 92.3 & 52.5 & 135.2 & 89.3 & 110.5 & 6.9 & 2174.0 & 196.5 & 139.5 & 1412.1 & 200.0 & 0.15 & 39.3 & 16.6 & 0.43 & 3.34 \\
\hline $\operatorname{Mean}_{2}$ & 92.3 & 51.2 & 130.9 & 85.5 & 106.9 & 6.9 & 1893.9 & 189.7 & 134.3 & 1212.9 & 183.3 & 0.14 & 38.7 & 16.3 & 0.42 & 2.90 \\
\hline $\mathbf{P}_{2}$ & NS & NS & $<0.005$ & $<0.001$ & $<0.001$ & $<0.025$ & NS & NS & NS & NS & NS & NS & NS & NS & NS & NS \\
\hline $\mathbf{P}_{\mathbf{3}}$ & NS & NS & 0.01 & $<0.005$ & 0.005 & $<0.05$ & NS & NS & NS & NS & NS & NS & NS & NS & NS & NS \\
\hline
\end{tabular}

The abbreviations are the same as those used in Table II. 
Table IV. Comparison of Resting and Exercise Hemodynamics in Normal and Hypertensive Subjects

\begin{tabular}{|c|c|c|c|c|c|c|c|c|c|c|c|c|c|c|c|c|}
\hline & HR & SV & $\mathrm{PAO}_{3}$ & $\mathrm{PAO}_{\mathrm{d}}$ & $\mathrm{PAo}_{\mathrm{m}}$ & LVEDP & $R$ & $Z_{1}$ & $Z_{\mathrm{c}}$ & $W_{\mathrm{t}}$ & $w_{0}$ & $W_{0} / W_{t}$ & $P_{\mathrm{f}}$ & $P_{\mathrm{b}}$ & $P_{\mathrm{b}} / P_{\mathrm{f}}$ & $f_{0}$ \\
\hline \multicolumn{17}{|c|}{ Resting: normal subjects $(n=6)$} \\
\hline Mean & 88.4 & 58.8 & 116.9 & 79.3 & 97.4 & 9.7 & 1669.0 & 150.4 & 119.8 & 1354.0 & 178.7 & 0.15 & 36.9 & 16.1 & 0.43 & 3.00 \\
\hline SD & 15.4 & 20.5 & 8.3 & 12.2 & 9.4 & 4.5 & 513.4 & 88.4 & 71.4 & 720.8 & 56.0 & 0.02 & 7.0 & 6.0 & 0.12 & 0.33 \\
\hline \multicolumn{17}{|c|}{ Exercise: normal subjects } \\
\hline Mean & 97.6 & 54.5 & 138.4 & 92.2 & 113.8 & 12.6 & 1854.0 & 191.4 & 138.6 & 1581.0 & 217.1 & 0.15 & 41.1 & 16.7 & 0.41 & 3.31 \\
\hline SD & 17.9 & 14.2 & 12.5 & 14.0 & 11.8 & 5.9 & 540.2 & 105.9 & 90.7 & 575.3 & 68.5 & 0.02 & 9.6 & 5.2 & 0.08 & 0.40 \\
\hline$\Delta(R-E)_{1}$ & -9.3 & 4.3 & -21.5 & -12.9 & -16.4 & -3.0 & -185.0 & -41.0 & -18.8 & -227.3 & -38.4 & 0.002 & -4.2 & -0.5 & 0.02 & -0.31 \\
\hline SD & 4.2 & 11.3 & 6.3 & 5.4 & 5.3 & 2.4 & 242.8 & 28.4 & 20.3 & 214.5 & 34.0 & 0.01 & 3.4 & 2.2 & 0.05 & 0.48 \\
\hline$P$ & 0.003 & NS & $<0.001$ & 0.002 & $<0.001$ & 0.03 & NS & 0.017 & 0.073 & 0.049 & 0.039 & NS & 0.03 & NS & NS & NS \\
\hline \multicolumn{17}{|c|}{ Resting: hypertensive subjects $(n=9)$} \\
\hline Mean & 80.7 & 58.1 & 162.6 & 96.6 & 126.8 & 16.3 & 2504.0 & 327.2 & 164.8 & 1565.0 & 238.7 & 0.16 & 49.1 & 26.3 & 0.54 & 3.86 \\
\hline SD & 5.3 & 22.5 & 18.8 & 12.2 & 13.8 & 4.4 & 1061.0 & 175.3 & 76.4 & 597.6 & 97.5 & 0.02 & 6.5 & 4.4 & 0.09 & 0.84 \\
\hline \multicolumn{17}{|c|}{ Exercise: hypertensive subjects } \\
\hline Mean & 87.5 & 60.1 & 190.6 & 109.4 & 145.6 & 19.2 & 2564.0 & 389.5 & 178.5 & 2085.0 & 334.8 & 0.16 & 57.5 & 30.8 & 0.54 & 4.04 \\
\hline SD & 6.3 & 25.3 & 29.5 & 14.4 & 18.9 & 4.6 & 989.9 & 196.8 & 67.8 & 1096.0 & 208.4 & 0.02 & 12.1 & 6.5 & 0.08 & 1.22 \\
\hline$\Delta(\mathrm{R}-\mathrm{E})_{2}$ & -6.8 & -1.9 & -28.0 & -12.9 & -18.7 & -2.9 & -59.7 & -62.3 & -13.7 & -519.6 & -96.1 & -0.003 & -8.5 & -4.5 & 0.0002 & -0.18 \\
\hline SD & 4.7 & 6.5 & 21.1 & 9.2 & 13.4 & 2.2 & 319.7 & 60.3 & 25.9 & 569.9 & 121.7 & 0.01 & 7.2 & 3.0 & 0.05 & 0.58 \\
\hline$P$ & 0.002 & NS & 0.004 & 0.003 & 0.003 & 0.005 & NS & 0.015 & NS & 0.026 & 0.045 & NS & 0.008 & 0.002 & NS & NS \\
\hline$P\left(\Delta_{1}\right.$ vs $\left.\Delta_{2}\right)$ & NS & NS & NS & NS & NS & NS & NS & NS & NS & NS & NS & NS & NS & 0.015 & NS & NS \\
\hline
\end{tabular}

in total external power. Like the response to exercise prior to administration of propranolol, exercise after propranolol thus did not further affect the reflections, pulse wave velocity, or compliance properties of the vasculature in the hypertensive
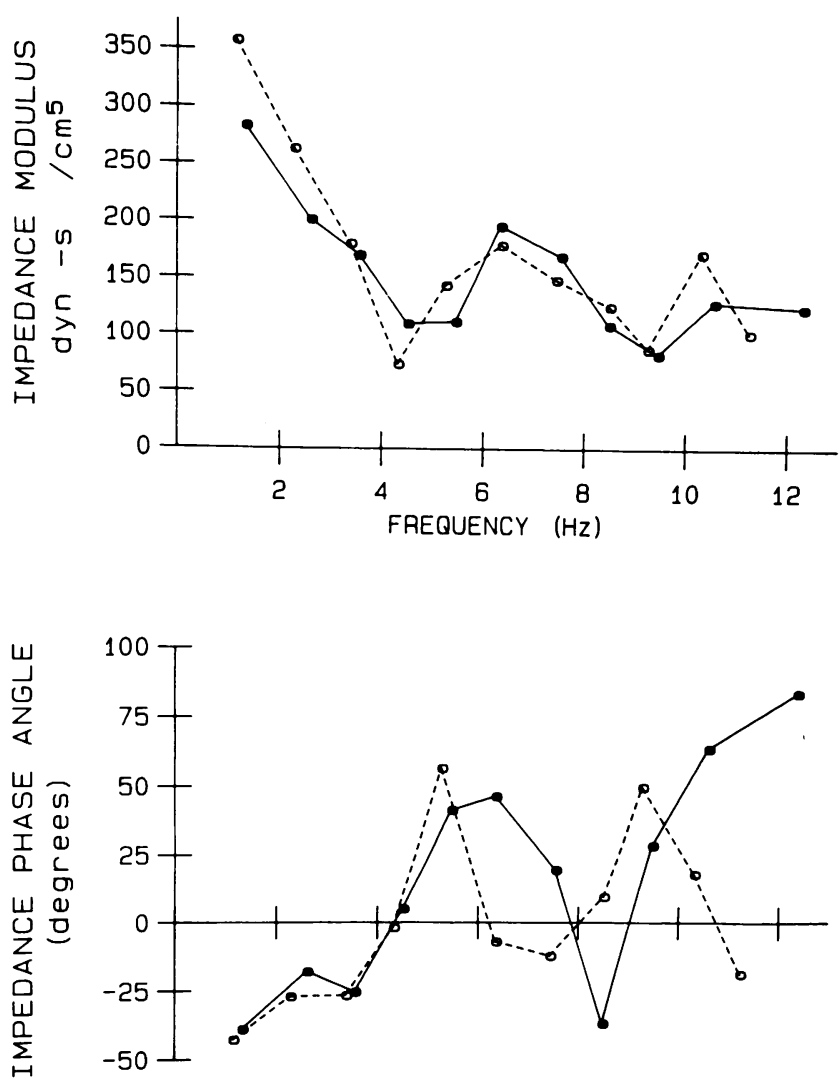

Figure 2. Averaged resting impedance moduli and phase angle spectra for the hypertensive subjects before (solid symbols and line) and after beta blockade with propranolol (open symbols and dotted line). group. Rather, the combined stress of exercise and beta blockade produced enough additional increase in the steady component of the hydraulic load to decrease forward output.

\section{Discussion}

Comparisons with other studies. Before discussing the implications of our findings it is important to put our study into perspective with previous studies. First, our normal and hypertensive groups did not differ significantly in age. If anything, the hypertensive patients tended to be somewhat younger than those with normal blood pressure. This is in contrast to the two previous studies of aortic impedance in human hypertension $(3,4)$ in which the hypertensive group in each case was significantly older than the normal or control group. This difference in study populations is important in view of studies indicating that aging itself may be associated with distinct alterations in the aortic impedance spectrum (13). We feel that the likelihood of significant atherosclerotic disease in our subjects is also quite low since the incidence of this disease process is low to begin with in Orientals living in the Far East. Furthermore, none of our subjects had any history of angina pectoris or other symptoms suggestive of coronary or other peripheral vascular disease. This is in contrast to the study of Nichols et al. (3) in which the hypertensive patients all had angiographically documented coronary artery disease. Although coronary vascular disease may or may not reflect the status of the remainder of the vasculature, interpretation of impedance must be qualified in a setting where the properties of the aortic wall could be significantly altered by a disease other than the one under study. Finally, since all of our subjects were Chinese, the possible confounding effect of racial heterogeneity was not a consideration.

Even though we examined a different racial group than in previous studies, the resting aortic impedance spectrum in our normal population was similar to those reported by others $(4,17-19,32)$. The impedance modulus fell steeply from its value of resistance and oscillated about the characteristic 
Table V. Acute Hemodynamic Response to Intravenous Propranolol in Hypertensive Subjects

\begin{tabular}{|c|c|c|c|c|c|c|c|c|c|c|c|c|c|c|c|c|}
\hline & HR & sv & PAO, $_{\mathbf{q}}$ & $\mathrm{PAO}_{\mathrm{d}}$ & $\mathbf{P A O}_{\mathbf{m}}$ & LVEDP & $R$ & $Z_{1}$ & $Z_{c}$ & $W_{1}$ & $W_{0}$ & $W_{0} / W_{t}$ & $P_{\mathrm{f}}$ & $P_{\mathrm{b}}$ & $P_{\mathrm{b}} / P_{\mathrm{f}}$ & $f_{0}$ \\
\hline \multicolumn{17}{|c|}{ Before propranolol $(n=10)$} \\
\hline Mean & 79.3 & 60.9 & 156.0 & 94.4 & 121.9 & 12.0 & 2370.0 & 284.0 & 147.8 & 1592.0 & 243.7 & 0.15 & 45.3 & 23.7 & 0.52 & 4.29 \\
\hline SD & 8.9 & 24.5 & 22.9 & 12.7 & 15.4 & 3.4 & 985.0 & 149.9 & 62.8 & 791.9 & 135.7 & 0.02 & 7.2 & 5.4 & 0.09 & 1.20 \\
\hline \multicolumn{17}{|c|}{ After propranolol } \\
\hline Mean & 68.1 & 59.5 & 166.0 & 98.3 & 126.7 & 16.6 & 2848.0 & 358.4 & 150.6 & 1360.0 & 208.0 & 0.15 & 46.8 & 27.7 & 0.60 & 3.97 \\
\hline SD & 6.6 & 21.9 & 25.3 & 12.9 & 16.3 & 4.2 & 1056.0 & 187.2 & 67.2 & 597.9 & 104.4 & 0.02 & 8.9 & 6.2 & 0.11 & 0.94 \\
\hline$\Delta(B-A)$ & 11.2 & 1.4 & -10.1 & -3.9 & -4.7 & -4.6 & -478.4 & -74.5 & -2.8 & 232.6 & 35.7 & 0.00 & -1.5 & -4.0 & -0.08 & 0.33 \\
\hline SD & 6.8 & 7.0 & 10.7 & 2.8 & 5.2 & 3.3 & 280.8 & 52.5 & 20.5 & 276.7 & 66.7 & 0.02 & 5.5 & 4.2 & 0.09 & 0.80 \\
\hline$P$ & $<0.001$ & NS & 0.016 & 0.002 & 0.018 & 0.002 & $<0.001$ & 0.002 & NS & 0.026 & NS & NS & NS & 0.014 & 0.022 & NS \\
\hline
\end{tabular}

$\Delta(B-A)$, change from before to after propranolol.

impedance $Z_{\mathrm{c}}$. Both the raw mean value for $Z_{\mathrm{c}}$ of 112 dyn-s/ $\mathrm{cm}^{5}$ and the more reliable weighted mean value for $Z_{\mathrm{c}}$ of 94 were close to the values ranging from 47 to 90 reported earlier in other normal populations. This disparity of estimates of characteristic impedance is not surprising in view of the potential sources of error involved in calculation. First, harmonic analysis yields only a limited number of harmonics to average. The low signal-to-noise ratio, especially in the higher harmonics of the flow signal further adds to the scatter. Finally, there is disagreement as to the initial frequency from which to begin averaging. Some authors use $2 \mathrm{~Hz}$ and others use 4 or $5 \mathrm{~Hz}$ as the initial frequency from which to begin the averaging $(3,4,16,17)$. In our study $Z_{\mathrm{c}}$ was $\sim 5 \%$ of resistance. This compares favorably with values ranging from 4 to $8 \%$ found in these earlier studies. The first zero crossing of the phase angle in our study was 3.0 $\mathrm{Hz}$, which is somewhat lower than the values of 3.1 to 4.3 previously reported. Since linear extrapolation was used to estimate the zero crossing, this small difference among the various studies is probably not significant. We thus feel that our normal population was comparable to those of other laboratories.

The abnormalities in the impedance spectrum in our hypertensive population, with some exceptions as denoted below, were in general agreement with the findings of previous studies $(3,4)$. These studies all demonstrated an elevated resistance, a rightward shift of the $f_{0}$ and an elevation in low frequency components of the spectrum. In very early essential hypertension, the earliest hemodynamic abnormalities are an elevated cardiac output with a normal resistance (5-7). The mechanism for the elevation of cardiac output is not clearly established but appears to be due primarily to an elevated heart rate (22), possibly on a neurogenic basis. In chronic essential hypertension cardiac out- put either returns to normal or falls below normal while peripheral resistance becomes elevated (8-10). Since we found that heart rate and cardiac output were not elevated but that resistance was increased, the patients in our hypertensive population, despite their relatively young age, should properly be categorized with chronic hypertension.

There are, however, some differences between our results and those previously described. First, Merillon et al. (4) found a higher than normal ratio of oscillatory to steady power in their hypertensive population. In six of their hypertensive patients given nitroprusside, all the impedance abnormalities normalized except that the elevated percentage of oscillatory power persisted. They attributed this persistent elevation in pulsatile power to either a remaining stiffness of the aortic wall or a more pulsatile flow wave becoming manifest after vasodilation. In contrast, we found no difference between the groups in the fraction of oscillatory power either before or after nitroprusside. We have no clear explanation for the differences in our results compared with theirs other than the small numbers of patients examined in that study. In fact, the ratio of oscillatory to mean power after administration of nitroprusside decreased in five of their six patients. Had more subjects been studied, their conclusions might have been different.

Second, Merillon et al. (4) did not find characteristic impedance to be elevated in their hypertensive group as compared to their normals. In contrast, Nichols et al. (3) found an elevated characteristic impedance in both their normotensive and hypertensive groups with coronary artery disease as compared with normal subjects without coronary artery disease. The number of subjects in this latter study, however, was small so that no statistical analysis of the data was performed. We found, in

Table VI. Hemodynamic Response to Handgrip Exercise after Propranolol in Hypertensive Subjects

\begin{tabular}{|c|c|c|c|c|c|c|c|c|c|c|c|c|c|c|c|c|}
\hline & HR & SV & PAo, & PAOd $_{d}$ & $\mathbf{P A o}_{\mathrm{m}}$ & LVEDP & $R$ & $Z_{1}$ & $Z_{c}$ & $W_{t}$ & $W_{0}$ & $W_{0} / W_{t}$ & $P_{\mathrm{f}}$ & $P_{\mathrm{b}}$ & $P_{\mathrm{b}} / P_{\mathrm{f}}$ & $f_{0}$ \\
\hline \multicolumn{17}{|c|}{ Resting $(n=9)$} \\
\hline Mean & 68.8 & 57.8 & 162.6 & 96.7 & 124.5 & 16.3 & 2882.0 & 364.8 & 157.2 & 1313.0 & 200.3 & 0.15 & 46.3 & 26.7 & 0.59 & 3.90 \\
\hline SD & 6.6 & 22.5 & 24.4 & 12.5 & 15.7 & 4.3 & 1115.0 & 197.4 & 67.7 & 614.3 & 107.7 & 0.02 & 9.3 & 5.7 & 0.11 & 0.98 \\
\hline \multicolumn{17}{|l|}{ Exercise } \\
\hline Mean & 75.0 & 52.8 & 183.9 & 106.6 & 138.9 & 19.9 & 3350.0 & 454.9 & 174.7 & 1485.0 & 230.0 & 0.16 & 52.3 & 30.7 & 0.60 & 4.07 \\
\hline SD & 6.1 & 22.0 & 29.9 & 15.7 & 19.2 & 4.9 & 1524.0 & 266.9 & 76.0 & 744.5 & 126.2 & 0.03 & 11.4 & 7.0 & 0.12 & 1.13 \\
\hline$\Delta(R-E)$ & -6.2 & 5.0 & -21.2 & -9.9 & -14.4 & -3.6 & -467.9 & -90.1 & -17.6 & -172.0 & -29.7 & -.007 & -6.0 & -4.0 & -0.01 & -0.16 \\
\hline SD & 5.5 & 5.0 & 14.1 & 6.1 & 8.4 & 1.9 & 480.2 & 85.2 & 25.3 & 242.3 & 53.1 & 0.02 & 6.3 & 2.9 & 0.06 & 0.70 \\
\hline$P$ & 0.009 & 0.017 & 0.002 & 0.001 & $<0.001$ & $<0.001$ & 0.019 & 0.013 & 0.071 & 0.066 & NS & NS & 0.022 & $<0.003$ & NS & NS \\
\hline
\end{tabular}


Table VII. Changes from Rest to Exercise in Hypertensive Subjects before and after Propranolol

\begin{tabular}{|c|c|c|c|c|c|c|c|c|c|c|c|c|c|c|c|c|}
\hline & HR & sv & $\mathrm{PAO}_{4}$ & $\mathrm{PAO}_{\mathrm{d}}$ & $\mathrm{PAo}_{\mathrm{m}}$ & LVEDP & $R$ & $Z_{1}$ & $Z_{\mathrm{c}}$ & $W_{\mathrm{t}}$ & $W_{0}$ & $W_{0} / W_{t}$ & $P_{\mathrm{f}}$ & $P_{\mathrm{b}}$ & $P_{\mathrm{b}} / P_{\mathrm{f}}$ & $f_{0}$ \\
\hline \multicolumn{17}{|c|}{ Before propranolol $(n=9)$} \\
\hline Mean $\Delta_{1}$ & -6.8 & -1.9 & -28.0 & -12.9 & -18.7 & -2.9 & -59.7 & -62.3 & -13.7 & -519.6 & -96.0 & -.003 & -8.5 & -4.5 & .0002 & -0.09 \\
\hline SD & 4.7 & 6.5 & 21.1 & 9.2 & 13.4 & 2.2 & 319.7 & 60.3 & 25.9 & 569.9 & 121.7 & 0.01 & 7.2 & 3.0 & 0.05 & 0.58 \\
\hline \multicolumn{17}{|c|}{ After propranolol $(n=9)$} \\
\hline Mean $\Delta_{2}$ & -6.2 & 5.0 & -21.2 & -9.9 & -14.4 & -3.6 & -467.9 & -90.1 & -17.6 & -172.0 & -29.7 & -.007 & -6.0 & -4.0 & -0.01 & 0.13 \\
\hline SD & 5.5 & 5.0 & 14.1 & 6.1 & 8.4 & 1.9 & 480.2 & 85.2 & 25.3 & 242.3 & 53.1 & 0.02 & 6.3 & 2.9 & 0.06 & 0.41 \\
\hline$\Delta\left(\Delta_{1}-\Delta_{2}\right)$ & -0.6 & -6.9 & -6.7 & -3.0 & -4.4 & 0.7 & 408.2 & 27.8 & 3.8 & -347.7 & -66.3 & 0.003 & -2.5 & -0.5 & 0.01 & -0.21 \\
\hline SD & 7.6 & 8.9 & 13.9 & 7.0 & 9.4 & 2.9 & 591.0 & 68.7 & 22.5 & 459.9 & 113.2 & 0.03 & 7.3 & 2.5 & 0.06 & 0.87 \\
\hline$P$ & NS & 0.049 & NS & NS & NS & NS & 0.072 & NS & NS & 0.053 & NS & NS & NS & NS & NS & NS \\
\hline
\end{tabular}

agreement with the data of Nichols et al. (3), that the characteristic impedance was higher in the group with hypertension than in our normal group. As discussed above, this parameter is quite difficult to determine accurately so further studies using different techniques may be required to unequivocally delineate how hypertension affects characteristic impedance.

Implications of our findings. In addition to confirming the hemodynamic changes described by previous studies, we found an increase in the ratio of backward to forward components of the pressure wave in the hypertensive group. This indicates an increased magnitude of peripheral reflections in hypertension. O'Rourke $(1,2)$ suggested this possibility based on some animal work, and Merillon et al. (4) interpreted their findings as indicating increased reflections. As far as we know, however, ours is the first direct evidence of increased wave reflections in human hypertension.

While it is clear that wave reflections can affect the contour of the pressure and flow waves $(19,33)$, the consequences of increased wave reflection on the heart have not been documented. Specifically, how reflected waves affect the heart depends upon both their amplitude and timing. Large elevated reflections together with a low pulse wave velocity, such as found in children or in some animals (34), may augment aortic diastolic pressure and thereby increase diastolic coronary artery driving pressure, which would be beneficial to the heart. In contrast, with both increased magnitude and increased pulse wave velocity, the reflections are likely to be manifested during ventricular systole and produce an additional load on the ejecting heart. How much this small pressure effect influences ventricular ejection is not clear. Recent simulation studies from our laboratory using data from senescent beagle dogs, support the possibility of a deleterious effect of early, large reflections on stroke volume (35). In a heart ejecting from the same end-diastolic volume to the same mean end-systolic pressure, the stroke volume was a few percent lower for the beat associated with increased wave reflection and pulse wave velocity than one with less reflection. It is intriguing to speculate that the long-term combined effect of increased reflections and pulse wave velocity may play a role in the cardiac sequelae of hypertensive heart disease.

It is generally considered that resistance and wave reflections are associated. That is, when resistance decreases, the amount of reflections decreases and vice versa. Our data, however, also indicate that this relationship may not always be concordant. After beta blockade both the resistance and wave reflections increased. Adding handgrip exercise to beta blockade further increased resistance but did not increase wave reflections. One explanation for this finding is that handgrip exercise produces a localized increase in resistance (26) that is not enough to be manifested in the normal state by increase in either wave reflections or total resistance. After beta blockade, however, with the unmasked generalized vasoconstriction, the additional resistance in the upper extremities is manifested as an increase in total peripheral resistance but there is still not enough alteration in the vasculature to further increase wave reflections. These findings are supportive of the concept that the design of the human vasculature is akin to an asymmetric T-tube (36), in which the predominant reflecting site is at the lower rather than at the upper extremities. Without further careful localized measurements, however, this concept remains speculative.

The responses to handgrip exercise and beta adrenergic blockade indicate that the resting abnormalities in wave reflection in the hypertensive group cannot be attributed solely to the elevated blood pressure. While exercise increased blood pressure it resulted in no further alteration in wave reflection. In contrast, compared to handgrip, beta blockade by itself resulted in much smaller increases in aortic pressures and yet produced a significant increase in wave reflection. Moreover, when pressure was further elevated with handgrip after beta blockade there was no further increase in wave reflections.

The fact that nitroprusside normalized all the hemodynamic parameters except for a slight residual elevation in blood pressure suggests that these abnormalities are related to smooth musclemediated increase in vascular tone. This increased tone is further unmasked during generalized beta adrenergic blockade but the exact mechanisms responsible for this elevated vascular tone cannot be determined from our results. Many previous studies have found that beta blockade affects hemodynamics in hypertension (22-27). For example, Ulrych et al. (23) found that intravenous beta blockade with propranolol did not acutely decrease blood pressure but rather increased peripheral resistance. Similarly, Hoel et al. (21) found that intravenous propranolol did not significantly lower blood pressure but increased resistance. With chronic oral propranolol both the blood pressure and resistance decreased. They suggested that the late decrease of resistance was due to a resetting of the baroreceptor reflexes. Julius et al. (27) found that elevated resting cardiac outputs and heart rates in a group of patients with borderline hypertension were partially normalized after propranolol infusion but were only completely normalized when atropine was added to the beta blockade. More recently, Levenson et al. (25) found that intravenous propranolol increased resistance and decreased estimated aortic compliance in older but not younger hypertensive patients. Finally, Levenson et al. (26) demonstrated that both selective and nonselective (propranolol) beta blockade produced 
an increase in total peripheral resistance, whereas only selective beta blockade produced an increase in forearm resistance and a decrease in forearm blood flow. Because the large vessel calibers were normal in the hypertensive group, they concluded that there was either a higher degree of vascular tone or there was decreased distensibility in this group.

The rightward shift of the impedance spectrum in hypertension was predicted by O'Rourke and was also found in the two previous studies in man (1-4). This shift is a manifestation of greater pulse wave velocity and has been observed in other instances, such as aging, where arterial stiffness is presumably increased (13). The increase in characteristic impedance is also compatible with increased aortic stiffness in the hypertensive group. This parameter is only an indirect index of aortic stiffness because of the competing effects of arterial size and stiffness on characteristic impedance. That is, with increasing muscle tone the vessel wall gets stiffer but its diameter may decrease. Depending upon where on the nonlinear pressure-volume relationship the vascular system is operating, the net effect of an increase in pressure could be no change, an increase, or a decrease in the calculated characteristic impedance.

Our study, like the two earlier ones in human hypertension $(3,4)$, also found an increase in the low-frequency harmonics of the impedance spectrum. Studies in animals (37) and in the three-element Windkessel model of the vasculature (38) show that an increase in the low frequency harmonic of impedance is a manifestation of either a decreased proximal aortic compliance or increased wave reflections. Because Fourier series analysis limits the amount of low frequency data to multiples of the fundamental frequency, we used the amplitude of $Z_{1}$ as an index of the low frequency portion of the impedance spectrum. An increase in $Z_{1}$ was seen in each instance that blood pressure increased even in cases where there were no increases in wave reflections. This suggests that the decrease in compliance is due to moving upward on a nonlinear portion of the aortic pressure-volume relationship. However, since we did not measure aortic compliance, we could not evaluate the separate contributions of decreased compliance and increased wave reflections to this increase in $Z_{1}$. Regardless, animal studies suggest that decreased compliance alone cannot be the primary mechanism for diastolic hypertension since a pure decrease in compliance would lower rather than increase diastolic aortic pressure (39).

With regard to therapeutic implications, although the exact mechanism by which beta blockade decreases blood pressure remains uncertain, it is an increasingly common mode of therapy. Our results show that peripheral reflections are increased after acute beta blockade. Whether this hemodynamic alteration occurs with chronic therapy and whether this would produce a deleterious long-term effect are not known. We also have not examined the detailed hemodynamic effects of other antihypertensive agents. Nevertheless, our study suggests that, especially in elderly patients in whom vascular abnormalities may already be present, treatment of the blood pressure alone, without consideration of other vascular consequences, may not be the ideal approach.

Limitations of our study. There are some limitations of our study that deserve further considerations. Our results pertain only to acute alterations induced by handgrip exercise and the pharmacological interventions employed in the catheterization laboratory. Clearly this setting may induce some anxiety and may not represent a true baseline condition. Further, how much the mild sedative affects the hemodynamic results is difficult to assess. Most importantly, some studies suggest that the acute and chronic effects of beta blockade on blood pressure differ (24). We found, as have other studies, that there was a slight increase in blood pressure after acute intravenous beta blockade $(23,40)$. Thus, we cannot predict how the impedance and reflections would be altered after chronic treatment with beta blockers where pressure would presumably be lowered.

From the standpoint of data analysis, use of standard Fourier analysis methods for calculating impedance limits the available data to discrete harmonics. This not only provides very little data in the low frequency range of the spectrum but, as discussed earlier, produces a certain degree of uncertainty in the calculations of characteristic impedance and the zero crossing of the phase angle. The fact that our findings generally confirm those of others, however, lends support to the validity of our data.

We have purposely not estimated values of compliance from our hemodynamic data. Many previous studies have calculated compliance by assuming that the diastolic aortic pressure decay is an exponential function of time (41). We recently critically examined the assumptions for this method underlying estimating compliance and concluded that any slight deviations from a monoexponential function would introduce large errors in the compliance estimates (42). We proposed a new method for estimating compliance but this approach depends upon the value of a parameter(s) derived from the pressure-volume relation of the aorta. Since we do not know how this parameter(s) is modified with hypertension or with the different interventions employed in the current study, we feel that further work needs to be completed before the method can be applied to data such as these.

In summary, our results demonstrate distinct differences from normal in the arterial hemodynamics of patients with hypertension. In addition to the well-documented higher arterial resistance, the aortic impedance at rest is characterized by a larger modulus of the first harmonic, higher characteristic impedance, a rightward shift of the entire spectrum, and greater wave reflections. These abnormalities are all eliminated with nitroprusside. During generalized beta blockade there are further increases in resistance, first modulus of impedance, and in wave reflections. These vascular abnormalities could not all be attributed to higher blood pressure. Rather, our data suggest that some of the altered hemodynamics in hypertensives can be attributed to an increased smooth muscle tone that is further unmasked during beta blockade.

\section{References}

1. O'Rourke, M. F. 1976. Pulsatile arterial hemodynamics in hypertension. Aust. N. Z. J. Med. (Suppl. II) 6:40-48.

2. O'Rourke, M. F. 1970. Arterial hemodynamics in hypertension. Circ. Res. (Suppl. II) 16 and 17:II-123-133.

3. Nichols, W. W., C. R. Conti, W. E. Walker, and W. R. Milnor. 1977. Input impedance of the systemic circulation in man. Circ. Res. 40:451-458.

4. Merillon, J. P., G. J. Fontenier, J. F. Lerallut, M. Y. Jaffrin, G. A. Motte, C. P. Genain, and R. R. Gourgon. 1982. Aortic input impedance in normal man and arterial hypertension: its modification during changes in aortic pressure. Cardiovasc. Res. 16:646-656.

5. Widimsky, J., M. H. Fejfarova, and Z. Fejfar. 1957. Changes of cardiac output in hypertensive disease. Cardiologia. 31:381-389.

6. Freis, E. D. 1960 . Hemodynamics of hypertension. Physiol. Rev. 40:27-54.

7. Eich, R. H., R. J. Peters, and R. P. Cuddy. 1962. The hemodynamics in labile hypertension. Am. Heart J. 63:188-195.

8. Guyton, A. C., and T. G. Coleman. 1969. Quantitative analysis 
of the pathophysiology of hypertension. Circ. Res. 24, 25(Suppl. 1):I-1I-19.

9. Lund-Johansen, P. 1977. Hemodynamic alterations in hypertension-spontaneous changes and effects of drug therapy. A review. Acta Med. Scand. (Suppl.) 603:1-14.

10. Kaplan, N. M. 1982. Primary (Essential) Hypertension. In Clinical Hypertension, Williams and Wilkins Co., Baltimore. 42-96.

11. Milnor, W. R. 1975. Arterial impedance as ventricular afterload. Circ. Res. 36:565-570.

12. Noble, M. I. M. 1979. Left ventricular load, arterial impedance and their interrelationship. Cardiovasc. Res. 13:183-198.

13. Nichols, W. W., M. F. O'Rourke, A. P. Avolio, T. Yaginuma, J. P. Murgo, C. J. Pepine, and C. R. Conti. 1985. Effects of age on ventricular-vascular coupling. Am. J. Cardiol. 55:1179-1184.

14. Pepine, C. J., W. W. Nichols, and C. R. Conti. 1978. Aortic input impedance in heart failure. Circulation. 58:460-465.

15. Pepine, C. J., W. W. Nichols, R. C. Curry, Jr., and C. R. Conti. 1979. Aortic input impedance during nitroprusside infusion. J. Clin. Invest. 64:643-654.

16. Yin, F. C. P., P. A. Guzman, K. P. Brin, W. L. Maughan, J. A. Brinker, T. A. Traill, J. L. Weiss, and M. L. Weisfeldt. 1983. Effect of nitroprusside on hydraulic vascular loads on the right and left ventricle of patients with heart failure. Circulation. 67:1330-1339.

17. Laskey, W. K., W. G. Kussmaul, J. L. Martin, J. P. Kleaveland, J. W. Hirshfeld, Jr., and S. Shroff. 1985. Characteristics of vascular hydraulic load in patients with heart failure. Circulation. 72:61-71.

18. Murgo, J. P., N. Westerhof, J. P. Giolma, and S. A. Altobelli. 1980. Effects of exercise on aortic input impedance and pressure wave forms in normal humans. Circ. Res. 48:334-343.

19. Murgo, J. P., N. Westerhof, J. P. Giolma, and S. A. Altobelli. 1981. Manipulation of ascending aortic pressure and flow wave reflections with the Valsalva maneuver: relationship to input impedance. Circulation. 63:122-132.

20. MacDonald, H. R., R. P. Sapru, S. H. Taylor, and K. W. Donald. 1966. Effect of intravenous propranolol on the systemic circulatory response to sustained handgrip. Am. J. Cardiol. 18:333-344.

21. Hoel, B. L., E. Lorentsen, and P. G. Lund-Larsen. 1970. Haemodynamic responses to sustained hand-grip in patients with hypertension. Acta Med. Scand. 188:491-495.

22. Julius, S., and M. Esler. 1975. Autonomic nervous cardiovascular regulation in borderline hypertension. Am. J. Cardiol. 36:685-696.

23. Ulrych, M., E. D. Frohlich, H. P. Dustan, and I. H. Page. 1968. Immediate hemodynamic effects of beta-adrenergic blockade with propranolol in normotensive and hypertensive man. Circulation. 37:411415.

24. Hansson, L., A. J. Zweifler, S. Julius, and S. N. Hunyor. 1974. Hemodynamic effects of acute and prolonged $\beta$-adrenergic blockade in essential hypertension. Acta Med. Scand. 196:27-34.

25. Levenson, J., A. Simon, B. Maarek, J. Totomoukouo, R. Gitelman, J. Bouthier, and M. Safar. 1983. Comparative effects of $\beta$-adrenergic blockade in systolic hypertension according to age. Eur. Heart J. 4(Suppl. G):51-54.
26. Levenson, J., A. C. Simon, M. E. Safar, J. D. Bouthier, and G. M. London. 1985. Elevation of brachial arterial blood velocity and volumic flow mediated by peripheral $\beta$-adrenoreceptors in patients with borderline hypertension. Circulation. 71:663-668.

27. Julius, S., A. V. Pascual, and R. London. 1971. Role of parasympathetic inhibition in the hyperkinetic type of borderline hypertension. Circulation. 44:413-418.

28. Brin, K. P., and F. C. P. Yin. 1984. Effect of nitroprusside on wave reflections in patients with heart failure. Ann. Biomed. Eng. 12: 135-150.

29. Westerhof, N., P. Sipkema, G. C. Van Den Bos, and G. Elzinga. 1972. Forward and backward waves in the arterial system. Cardiovasc. Res. 6:648-656.

30. Mosteller, M., and J. W. Tukey. 1977. Data Analysis and Regression. Addison-Wesley, Inc., Reading, MA. pp. 203-220.

31. Glantz, S. A. 1981. Primer of Biostatistics. McGraw-Hill, Book Co., New York, pp. 269-311.

32. Gundel, W., G. Cherry, B. Rajagopalan, L. Tan, G. Lee, and D. Schultz. 1981. Aortic input impedance in man: acute response to vasodilator drugs. Circulation. 63:1305-1314.

33. Van den Bos, G. C., N. Westerhof, G. Elzinga, and P. Sipkema. 1976. Reflection in the systemic arterial system: effects of aortic and carotid occlusion. Cardiovasc. Res. 10:565-573.

34. Avolio, A. P., W. W. Nichols, and M. F. O'Rourke. 1984. Exaggerated wave reflection in the kangaroo simulates arterial counterpulsation. Am. J. Physiol. 246:R267-R270.

35. Latson, T. W., F. C. P. Yin, and W. C. Hunter. 1986. The effects of finite wave velocity and discrete reflections on ventricular loading in Ventricular/Vascular Coupling: Clinical, Physiological and Engineering Aspects. F. Yin, editor. Springer-Verlag Inc., New York. 334-383.

36. O'Rourke, M. F. 1967. Pressure and flow waves in systemic arteries and the anatomical design of the arterial system. J. Appl. Physiol. 23: 139-149.

37. Westerhof, N., G. C. Van den Bos, and G. Elzinga. 1973. Influence of central and peripheral changes on the hydraulic input impedance of the systemic arterial tree. Med. Biol. Eng. 11:710-723.

38. Sunagawa, K., W. L. Maughan, and K. Sagawa. 1985. Stroke volume effect of changing arterial input impedance over selected frequency ranges. Am. J. Physiol. 17:H477-H484.

39. Randall, O. S., G. C. Van den Bos, and N. Westerhof. 1984. Systemic compliance: does it play a role in the genesis of essential hypertension? Cardiovasc. Res. 18:455-462.

40. Yin, F. C. P., G. S. Raizes, T. Guarnieri, H. A. Spurgeon, E. G. Lakatta, N. J. Fortuin, and M. L. Weisfeldt. 1978. Age-associated decrease in ventricular response to haemodynamic stress during beta-adrenergic blockade. Br. Heart J. 40:1349-1355.

41. Simon, A. Ch., M. E. Safar, J. A. Levenson, G. M. London, B. I. Levy, and N. P. Chau. 1979. An evaluation of large arteries compliance in man. Am. J. Physiol. 237(5):H550-H554.

42. Liu, Z. R., K. P. Brin, and F. C. P. Yin. 1985. Estimation of arterial compliance: an improved method and evaluation of current methods. Am. J. Physiol. 251(3):H588-H600. 